\title{
Solvent-free chromium catalyzed aerobic oxidation of biomass-based alkenes as a route to valuable fragrance compounds
}

\author{
Patricia A. Robles-Dutenhefner ${ }^{a}$, Bruno B.N.S. Brandão ${ }^{b}$, Líniker F. de Sousa ${ }^{a}$, Elena V. Gusevskaya ${ }^{b, *}$ \\ a Departamento de Química, Universidade Federal de Ouro Preto, 35400-000 Ouro Preto, MG, Brazil \\ b Departamento de Química, Universidade Federal de Minas Gerais, 31270-901 Belo Horizonte, MG, Brazil
}

\section{A R T I C L E I N F O}

\section{Article history:}

Received 24 January 2011

Received in revised form 21 March 2011

Accepted 28 March 2011

Available online 1 April 2011

\section{Keywords:}

Monoterpenes

Oxidation

Oxygen

Chromium catalysts

Mesoporous molecular sieves

\begin{abstract}
A B S T R A C T
Chromium containing mesoporous molecular sieves MCM-41 were shown to be an efficient heterogeneous catalyst for the liquid-phase aerobic oxidation of various monoterpenic alkenes under mild solvent-free conditions. The material was prepared through a direct hydrothermal method and characterized by ICP-AES, $\mathrm{N}_{2}$ adsorption-desorption, TEM, XRD, SAXS, and $\mathrm{H}_{2}$-TPR techniques. Characterizations suggest that chromium introduced in MCM-41 is essentially incorporated in the silica framework, with no extraframework chromium oxides being detected. Various oxygenated monoterpenoids important for the flavor and fragrance industry were obtained with high combined selectivities (75-92\%) at 30-40\% substrate conversions. The oxidation of $\beta$-pinene led almost exclusively to allylic mono-oxygenated derivatives, whereas limonene and $\alpha$-pinene gave both epoxides and allylic oxidation products. The catalyst undergoes no metal leaching and can be easily recovered and re-used. A silica-included chromium catalyst prepared through a conventional sol-gel method showed activity comparable with that of $\mathrm{Cr}$ MCM-41; however, selectivity was much lower.
\end{abstract}

(c) 2011 Elsevier B.V. All rights reserved.

\section{Introduction}

The catalytic oxidation of organic compounds with molecular oxygen represents an attractive route to a great variety of valuable commodity and specialty chemicals. These processes occur with high atom efficiency giving water as the only byproduct and can replace conventional stoichiometric processes in the fine chemicals industry [1]. In the case of liquid-phase oxidations, the development of the technologies which use solid catalysts under solvent-free conditions is particularly relevant to green chemistry concepts.

A conventional route to prepare heterogeneous metal catalysts is the impregnation of metal compounds onto the solid surface. A general problem associated with the use of such catalysts in liquid-phase oxidations is a rapid leaching of metal ions from the solid support. On the other hand, the application of heterogeneous gas-phase processes in the fine chemicals industry is restricted by limited volatility and thermal stability of complex substrates. A promising approach to develop stable catalysts for liquid-phase oxidations is the incorporation of redox-active metals in the framework positions of solid matrices during the synthesis of the solid,

\footnotetext{
* Corresponding author at: Universidade Federal de Minas Gerais, Departamento de Quimica, Av Antonio Carlos 6627, 31270-901 Belo Horizonte, Minas Gerais, Brazil. Tel.: +55 3134095741; fax: +55 3134095700 .

E-mail address: elena@ufmg.br (E.V. Gusevskaya).
}

i.e., isomorphic substitution [1,2]. Such site-isolation of active metal ions can be an important factor in catalytic liquid-phase oxidations in heterogeneous systems. It often results in the materials highly stable to leaching and also prevents the aggregation of the metal to less reactive species [1].

Ordered mesoporous molecular sieves, such as MCM-41, discovered by Mobil Company in 1992 have received considerable attention in catalysis research over the last decades [3,4]. The MCM-41 material possesses a high surface area (above $700 \mathrm{~m}^{2}$ ) and hexagonal array of uniform mesopores (2-10 nm) with narrow pore size distribution. Such large pore catalysts are especially important for the conversion of bulky molecules in the fine chemicals industry. No diffusion problems are usually observed with the catalysts based on MCM-41 [2].

Mesoporous molecular sieves containing different transition metals have a great potential as heterogeneous catalysts in liquidphase oxidations. We have recently developed an efficient process for the aerobic oxidation of isolongifolene, one of the most available sesquiterpenes, over Co-MCM-41 [5]. The catalyst was stable to leaching and exhibited much higher selectivity then the material prepared by a conventional sol-gel method without the surfactant. The aim of the present work was to prepare MCM-41-included chromium materials and to study their potential as redox catalysts for the aerobic oxidation of biomass-based alkenes.

Chromium reagents are widely used in organic chemistry in both stoichiometric and catalytic oxidations [6]. Considering environmental factors, the most attractive approach obviously consists 
in using toxic chromium compounds in catalytic amounts and in heterogenized forms. A number of chromium containing solid materials have been utilized in liquid-phase oxidations of organic substrates (mostly, alkanes and alkylaromatics) with $\mathrm{H}_{2} \mathrm{O}_{2}$, tertbutyl hydroperoxide (TBHP) [7-10], or molecular oxygen [11-13] as final oxidants. However, the main problem of these catalysts was chromium leaching out of the solid, especially in the presence of water [1].

In recent years, considerable attention has been focused on the development of chromium/MCM-41 catalysts for the liquid-phase oxidations of alkanes and aromatics with $\mathrm{H}_{2} \mathrm{O}_{2}$ or TBHP [14-20]. Among them, MCM-41-included materials, i.e., those prepared via the direct incorporation of chromium ions during the synthesis of the molecular sieve, exhibited remarkable stability even under aqueous conditions. In some of these works, Cr-MCM-41 materials maintained activity in several recycling experiments, with the reaction being stopped after catalyst removal $[16,18,19]$. In other studies $[17,20]$, loss of activity was observed only in the first recycling experiment due to leaching of non-framework chromium ions and then the activity remained nearly the same.

To the best of our knowledge no attempts to use MCM41-included chromium catalysts for the oxidation of organic compounds with molecular oxygen have been reported so far. Within our program aimed at adding value to natural ingredients of renewable essential oils, we decided to apply $\mathrm{Cr}-\mathrm{MCM}-41$ catalysts for the liquid-phase aerobic oxidation of limonene, $\alpha$-pinene, and $\beta$-pinene, the most abundant natural terpenes.

Terpenic compounds, in general, are an important renewable feedstock for the flavor and fragrance industry. Their oxygenated derivatives usually show interesting organoleptic properties and form one of the most important groups of fragrance ingredients [21]. The oxidation of $\alpha$-pinene is also relevant because verbenone, the product of the allylic oxidation of $\alpha$-pinene, is widely used for the synthesis of taxol, an important therapeutic agent. For several years, we have been interested in the aerobic oxidation of monoterpenic alkenes catalyzed by palladium [22-24] and cobalt [25-27] compounds. As concerned with chromium compounds, they are known to catalyze the allylic oxidation of alkenes by TBHP $[6-8,28]$. However, no heterogeneous chromium catalysts stable to leaching under the conditions of these reactions have been reported yet, as far as we know. The photocatalytic oxidation of alkenes with molecular oxygen on chromium containing silica gave both epoxide and allylic oxidation products $[29,30]$.

In the present work, we report a simple and efficient aerobic oxidation of various monoterpenes with high combined selectivities for valuable epoxy and/or allylic derivatives under mild solvent free conditions. The MCM-41 material containing chromium incorporated into the framework (Cr-MCM-41) was used as a heterogeneous, stable to leaching catalyst. The performance of $\mathrm{Cr}$ MCM-41 was compared with that of the silica-included chromium material prepared by a conventional sol-gel method without a surfactant.

\section{Experimental}

\subsection{Catalyst preparation and characterization}

The $5.0 \mathrm{wt} \% \mathrm{Cr} / \mathrm{SiO}_{2}$ catalyst (denoted as $\mathrm{Cr}-\mathrm{SiO}_{2} /$ sol-gel) was prepared by a sol-gel method using tetraethoxysilane ( $15.1 \mathrm{~g}$, TEOS, $98 \%$, Sigma-Aldrich) and $\mathrm{CrCl}_{3} \cdot 6 \mathrm{H}_{2} \mathrm{O}$ (1.0 g, Sigma-Aldrich) as precursors. The sol was obtained from a TEOS/ethanol/water mixture in a 1/3/10 molar ratio with the addition of $\mathrm{HCl}$ and $\mathrm{HF}$ (up to $\mathrm{pH} 2.0$ ) as catalysts. The sample was dried at $110^{\circ} \mathrm{C}$ for $24 \mathrm{~h}$ and thermally treated for $2 \mathrm{~h}$ at $900^{\circ} \mathrm{C}$ in air.
The 5.0 wt\% $\mathrm{Cr} / \mathrm{MCM}-41$ catalyst (denoted as $\mathrm{Cr}-\mathrm{MCM}-41$ ) was prepared by a direct incorporation of $\mathrm{Cr}$ into the MCM-41 framework aiming for the isomorphous substitution of $\mathrm{Si}$ by $\mathrm{Cr}$ ions. TEOS and $\mathrm{CrCl}_{3} \cdot 6 \mathrm{H}_{2} \mathrm{O}$ (Sigma-Aldrich) were used as precursors and hexadecyltrimethylammonium bromide (C16-TAB, Sigma-Aldrich) as a structure template. The $\mathrm{C} 16-\mathrm{TAB}$ solution in water was added to the solution of TEOS $(2.5 \mathrm{~g})$ in aqueous tetramethylammonium hydroxide (TMAOH, $25 \mathrm{wt} \%$, Sigma-Aldrich). The mixture was stirred for $30 \mathrm{~min}$ before $\mathrm{CrCl}_{3} \cdot 6 \mathrm{H}_{2} \mathrm{O}$ (1.6g) and remaining TEOS (21.0 g) were added. After additional mixing at $40^{\circ} \mathrm{C}$ for $24 \mathrm{~h}$, the mixture was placed in the autoclave at $100^{\circ} \mathrm{C}$ for $24 \mathrm{~h}$ and then cooled to room temperature. The obtained solid was separated by filtration, washed with de-ionized water and ethanol, and dried at $40^{\circ} \mathrm{C}$. Then, the solid was heated from room temperature to $550^{\circ} \mathrm{C}$ under flowing nitrogen and calcinated for $3 \mathrm{~h}$ at $550^{\circ} \mathrm{C}$ under flowing air to remove the residual organics. The TEOS/C16$\mathrm{TAB} / \mathrm{TMAOH} /$ water molar ratio was $1.0 / 0.12 / 0.3 / 22.0$.

The determination of total chromium contents was performed by inductively coupled plasma atomic emission spectrometry (ICP-AES) on a Spectro Ciros CCD instrument. The samples were dissolved in a mixture of $\mathrm{HF}$ and $\mathrm{HNO}_{3}$ before the measurements.

The textural characteristics of the catalysts were determined from nitrogen adsorption isotherms (Autosorb-Quantachrome NOVA-1200 instrument, nitrogen, $\left.-196^{\circ} \mathrm{C}\right)$. Samples were outgassed for $2 \mathrm{~h}$ at $300^{\circ} \mathrm{C}$ before analysis. Specific surface areas and average pore diameter were determined by the Brunauer-EmmettTeller (BET) equation. The pore size distributions were calculated from the desorption isotherms using the Barrett-Joyner-Halenda $(\mathrm{BJH})$ method. Average pore diameters were determined by the $\mathrm{BJH}$ method.

Transmission microscope (TEM) characterization was performed through a Tecnai-G2-20 (FEI) electron microscope with an acceleration potential of $200 \mathrm{kV}$.

The small angle X-ray scattering (SAXS) experiment at the D11ASAXS beam line was performed at the LNLS synchrotron laboratory, Campinas, Brazil using a Huber-423 3-circle diffractometer. The SAXS setup was equipped with a Si (1 111$)$ monochromator, giving a horizontally focused X-ray beam. The incident X-ray wavelength $\lambda$ was $1.488 \mathrm{~nm}$ and the scattering angle $2 \theta$ was approximately $0-10^{\circ}$.

The powder X-ray diffractometry (XRD) measurements were performed on a Rigaku model Geigerflex-3034 equipment using a $\mathrm{Co}(\mathrm{K} \alpha)$ radiation scanning from 4 to $70^{\circ}(2 \theta)$ to register the presence of chromium oxide phases.

The temperature-programmed reduction with hydrogen $\left(\mathrm{H}_{2}\right.$ TPR) was performed on a Quantachrome-ChemBET 300 instrument equipped with a thermal conductivity detector. The experiments were performed between 30 and $900^{\circ} \mathrm{C}\left(10^{\circ} \mathrm{C} / \mathrm{min}\right)$ in a flow of a $\mathrm{N}_{2} / \mathrm{H}_{2}$ mixture containing $5 \mathrm{vol} \%$ of $\mathrm{H}_{2}$.

\subsection{Catalytic oxidation experiments}

The reactions at atmospheric pressure were carried out in a glass reactor equipped with a magnetic stirrer and sampling system. The reactions at higher pressure were carried out in a stainless steel $20-\mathrm{mL}$ reactor (autoclave) equipped with a magnetic stirrer. In a typical run, a mixture of monoterpene $(6.2 \mathrm{mmol})$ and the catalyst $(0.03 \mathrm{~g}$, ca. $3.5 \mathrm{wt} \%)$ was transferred in the reactor. The glass reactor was connected to a gas burette containing molecular oxygen to measure a gas uptake. The autoclave was pressurized with molecular oxygen to the total pressure of $5 \mathrm{~atm}$. The reactors were placed in an oil bath; then, the solutions were intensively stirred at $60^{\circ} \mathrm{C}$ for the reported time. The reactions were followed by measuring an oxygen uptake and/or by gas chromatography (GC) using undecane as the internal standard (Shimadzu 17 instrument, Carbowax $20 \mathrm{M}$ capillary column). The aliquots were diluted fifteen-fold with acetonitrile before the GC analysis. The difference in mass balance 
Table 1

Elemental analysis data and textural properties of $\mathrm{Cr}-\mathrm{MCM}-41$ and $\mathrm{Cr}-\mathrm{SiO} / 2 /$ sol-gel.

\begin{tabular}{llllll}
\hline Sample & $\begin{array}{l}\mathrm{Cr} \\
\text { content } \\
(\mathrm{wt} \%)\end{array}$ & $\begin{array}{l}\text { BET } \\
\text { surface } \\
\text { area } \\
\left(\mathrm{m}^{2} \mathrm{~g}^{-1}\right)\end{array}$ & $\begin{array}{l}\text { Total } \\
\text { pore } \\
\text { volume } \\
\left(\mathrm{cm}^{3} \mathrm{~g}^{-1}\right)\end{array}$ & $\begin{array}{l}\text { BJH } \\
\text { average } \\
\text { pore } \\
\text { diameter } \\
(\mathrm{nm})\end{array}$ & Color \\
\hline $\mathrm{Cr}-\mathrm{MCM}^{-41}$ & 4.9 & 622 & 0.53 & 3.4 & Yellow \\
$\mathrm{Cr}-\mathrm{SiO}_{2} /$ sol-gel & 5.1 & 244 & 0.97 & 18.7 & Pale green \\
\hline
\end{tabular}

was attributed to the formation of oligomers, which were GC unobservable.

Catalyst recycling experiments were performed as follows: after the reaction, the catalyst was centrifuged, washed with acetonitrile and ethanol and reused. To control metal leaching, the catalyst was removed at the reaction temperature after $0.5 \mathrm{~h}$ and the solution was allowed to react further.

The structures of products 4-15 were confirmed by GC/MS (Shimadzu QP2010-PLUS instrument, $70 \mathrm{eV}$ ) by comparison with authentic samples. NMR and/or MS data for products 4-15 were presented in our previous paper [26].

\section{Results and discussion}

\subsection{Characterization of the catalysts}

The results of the elemental analysis of the prepared samples, their BET surface areas, average pore sizes, and pore volumes are presented in Table 1. Nitrogen adsorption-desorption isotherms and corresponding $\mathrm{BJH}$ pore size distributions are shown in Figs. 1 and 2. The $\mathrm{Cr}-\mathrm{MCM}-41$ material shows a much higher specific surface area $\left(622 \mathrm{~m}^{2} \mathrm{~g}^{-1}\right)$ than $\mathrm{Cr}-\mathrm{SiO}_{2} /$ sol-gel $\left(244 \mathrm{~m}^{2} \mathrm{~g}^{-1}\right)$ and a much smaller average BJH pore diameter ( $3.4 \mathrm{~nm}$ for Cr-MCM-41 vs. $18.7 \mathrm{~nm}$ for $\mathrm{Cr}-\mathrm{SiO}_{2} /$ sol-gel).

The Cr-MCM-41 sample exhibits a type IV isotherm (in the IUPAC classification) with a well defined $\mathrm{H} 1$ hysteresis loop at intermediate relative pressures, which indicate a textural mesoporosity with uniform cylindrical pore geometry and facile pore connectivity (Fig. 1) [31,32]. The pore size distribution in our Cr-MCM-41 sample is narrow and monomodal, showing a peak pore diameter at $3.4 \mathrm{~nm}$ with a peak width of ca. $2.4 \mathrm{~nm}$ at half-maximum (Fig. 1). Thus, the high BET surface area, nitrogen adsorption-desorption isotherm, and narrow pore size distribution confirm the uniform mesoporosity and organized structure of the $\mathrm{Cr}-\mathrm{MCM}-41$ sample.

The ordered hexagonal structure of $\mathrm{Cr}-\mathrm{MCM}-41$ was further confirmed by TEM (Fig. 3). Although the micrographs do not allow to determine the shape of individual pores, a regularity of pore

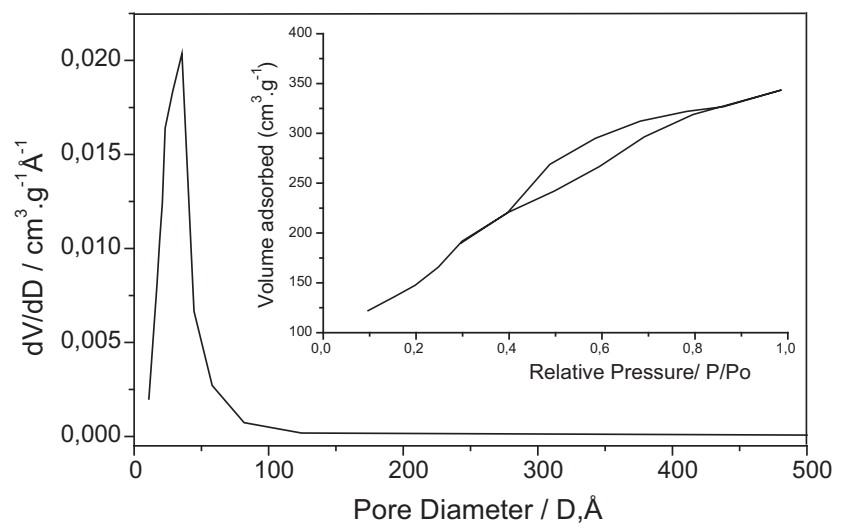

Fig. 1. Nitrogen adsorption-desorption isotherm for the Cr-MCM-41 sample and pore size distribution calculated from the desorption isotherm using the BJH method.

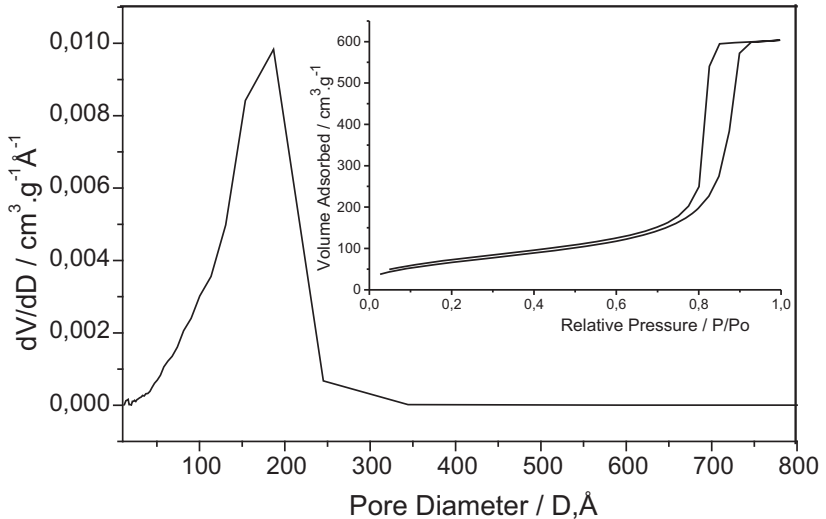

Fig. 2. Nitrogen adsorption-desorption isotherm for the $\mathrm{Cr}-\mathrm{SiO}_{2} /$ sol-gel sample and pore size distribution calculated from the desorption isotherm using the $\mathrm{BJH}$ method.

sizes can be clearly seen. In addition, the TEM images of Cr-MCM-41 unambiguously show the existence of the hexagonal arrangement of uniform pores (Fig. 3a).

The isotherm for the $\mathrm{Cr}-\mathrm{SiO}_{2} /$ sol-gel material with a sharp inflection step also corresponds to the type IV, which is characteristic of mesoporous materials (Fig. 2). The appearance of the hysteresis loop of type $\mathrm{H} 1$ at high relative pressures $\left(P / P_{0} \approx 0.7-0.9\right)$ may be related to the textural interparticle mesoporosity or macroporosity. A pore size distribution is also quite narrow and monomodal (Fig. 2). However, the average pore diameter in $\mathrm{Cr}-\mathrm{SiO}_{2} /$ sol-gel $(18.7 \mathrm{~nm})$ is much larger than that in $\mathrm{Cr}-\mathrm{MCM}-41$
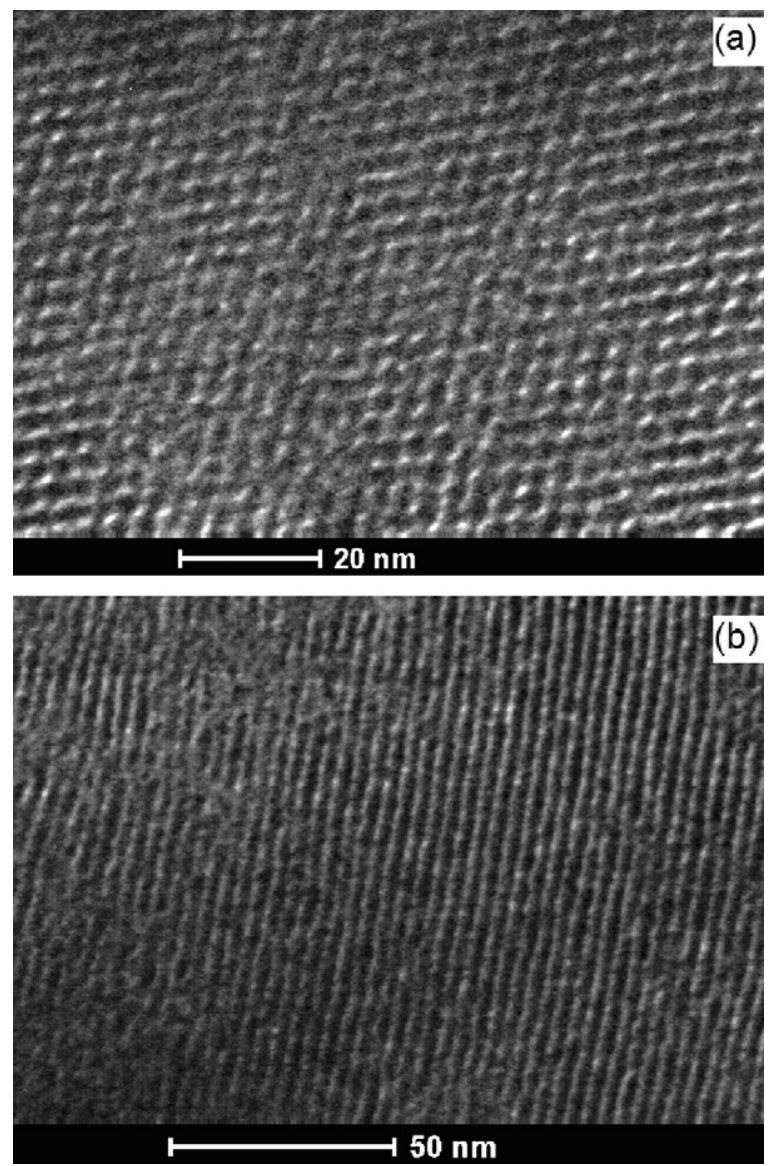

Fig. 3. HR-TEM micrograph of the Cr-MCM-41 sample: (a) perpendicular to the channel axis; (b) parallel to the main axis of the mesopores. 


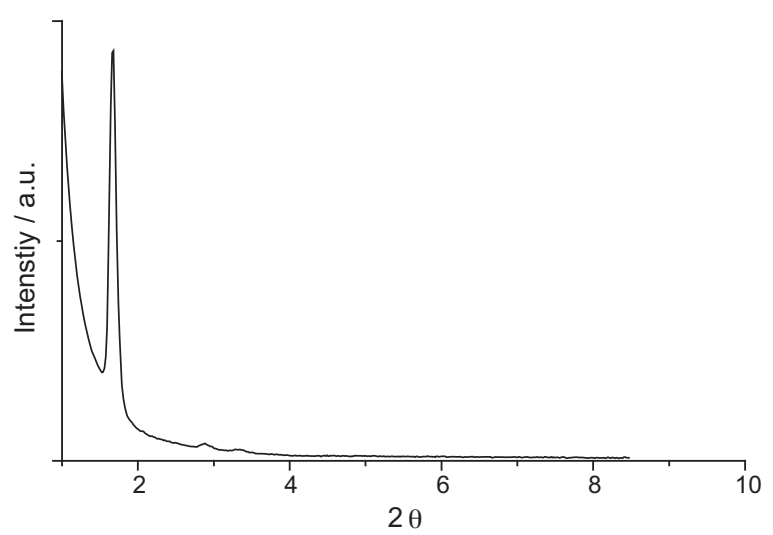

Fig. 4. SAXS pattern for the Cr-MCM-41 sample.

$(3.4 \mathrm{~nm})$. In addition, the $\mathrm{Cr}-\mathrm{SiO}_{2} /$ sol-gel material exhibits much broader pore size distribution than Cr-MCM-41 (cf. Fig. 2 and Fig. $1)$.

The structure of most ordered mesoporous materials shows a long range ordering of amorphous elements that can be studied by a SAXS technique. The synchrotron radiation small-angle pattern for the Cr-MCM-41 (Fig. 4) exhibits ( 100$)$ ), (1 110$)$ and (2 00 ) reflections at $2 \theta=1.67,2.90$ and $3.35^{\circ}$, respectively. The peaks can be indexed to the 2D hexagonal symmetry, indicating a highly ordered hexagonal structure [3]. Thus, the SAXS experiment has also confirmed that the prepared chromium-containing material has a typical structure of the MCM-41 silicate.

The diffractogram of the Cr-MCM-41 material exhibits no defined peaks of chromium oxide phases (Fig. 5a). On the other hand, the XRD pattern for $\mathrm{Cr}-\mathrm{SiO}_{2} /$ sol-gel (Fig. 5b) shows the presence of weak peaks characteristic of the $\mathrm{Cr}_{2} \mathrm{O}_{3}$ phase indicating the formation of small amounts of chromium oxide microcrystallites on the material surface. The crystallites size estimated from the most intense peak using the Scherrer equation showed the value of $26 \mathrm{~nm}$. The matrices of both materials are amorphous.

After calcinations, the $\mathrm{Cr}-\mathrm{SiO}_{2} /$ sol-gel material was pale green in color, whereas the Cr-MCM-41 sample was yellow. The green color could be due to the presence of $\mathrm{Cr}^{3+}$ ions, whereas the yellow one could be caused by $\mathrm{Cr}^{6+}$ ions. Really, the TPR profile of the Cr-MCM-41 sample (Fig. 6) exhibits a broad peak at $430^{\circ} \mathrm{C}$, which can be assigned to the reduction of $\mathrm{Cr}^{6+}$ to $\mathrm{Cr}^{3+}[33,34]$. On the other hand, the $\mathrm{Cr}-\mathrm{SiO}_{2} /$ sol-gel material shows only high temperature peaks: one at $665^{\circ} \mathrm{C}$ and the other at $870^{\circ} \mathrm{C}$ (Fig. 6). These peaks can be related to the reduction of strongly interacting with the silica matrix $\mathrm{Cr}^{3+}$ ions. In the TPR profile of $\mathrm{Cr}-\mathrm{MCM}-41$, there are no well-defined high temperature peaks up to $900^{\circ} \mathrm{C}$, suggesting even stronger interaction of $\mathrm{Cr}^{3+}$ ions with the silica matrix in this material than in $\mathrm{Cr}-\mathrm{SiO}_{2} /$ sol-gel. This fact is expected to contribute valuably to the stability of the catalyst toward metal

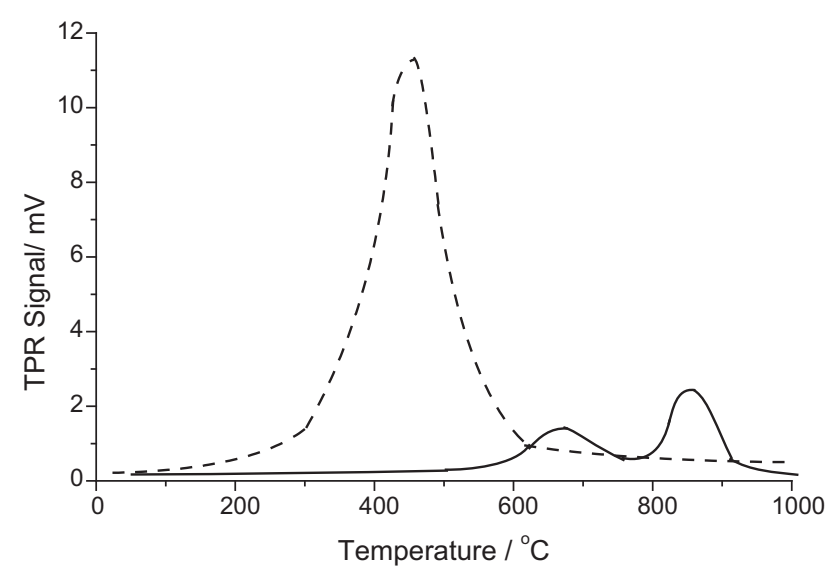

Fig. 6. TPR profiles of the $\mathrm{Cr}-\mathrm{MCM}-41$ (dotted line) and $\mathrm{Cr}-\mathrm{SiO}_{2} /$ sol-gel samples.

leaching under liquid-phase oxidative conditions. It should be mentioned that in the $\mathrm{H}_{2}$-TPR profiles of both samples, there are no low temperature peaks which could be assigned to the reduction of bulk $\mathrm{Cr}_{2} \mathrm{O}_{3}$ (expected at $250-300^{\circ} \mathrm{C}[34,35]$ ). Thus, the amounts of the extra-framework $\mathrm{Cr}_{2} \mathrm{O}_{3}$ in these materials should be small or even negligible, which correlates with the XRD data (Fig. 5).

The results obtained support the hypothesis that, at least in the Cr-MCM-41 sample, chromium seems to be essentially incorporated in the silica framework or attached to the surface via surface functional groups, e.g., hydroxyl groups. Small amounts of extraframework $\mathrm{Cr}_{2} \mathrm{O}_{3}$ were detected only in the $\mathrm{Cr}-\mathrm{SiO}_{2} /$ sol-gel material.

\subsection{Catalytic studies}

The behavior of the prepared $\mathrm{Cr}-\mathrm{SiO}_{2} /$ sol-gel and $\mathrm{Cr}-\mathrm{MCM}-41$ samples was examined in the oxidation of $\beta$-pinene (1), $\alpha$-pinene (2), and limonene (4) under solventless conditions. In all experiments, the reactions were carried out in neat liquid substrates under the atmosphere of molecular oxygen. The results are presented in Tables 2-4.

In blank reactions in the absence of catalysts, the conversions of all tested monoterpenes exposed to oxygen at $60^{\circ} \mathrm{C}$ were negligible, both at 1 and 5 atm (not shown in the Tables). On the other hand, both chromium containing materials promoted relatively rapid oxidations of these substrates showing similar activities but different selectivities. It is remarkable that the selectivities for the corresponding products obtained with $\mathrm{Cr}-\mathrm{MCM}-41$ were much higher than those obtained with $\mathrm{Cr}-\mathrm{SiO}_{2} /$ sol-gel. In general, the selectivities obtained with the $\mathrm{Cr}-\mathrm{MCM}-41$ material were the best or among the best reported so far in the literature [22-27]. The oxidation of limonene and $\beta$-pinene gave only four major products with a combined selectivity of $75-90 \%$ at $30-40 \%$ conversions, which
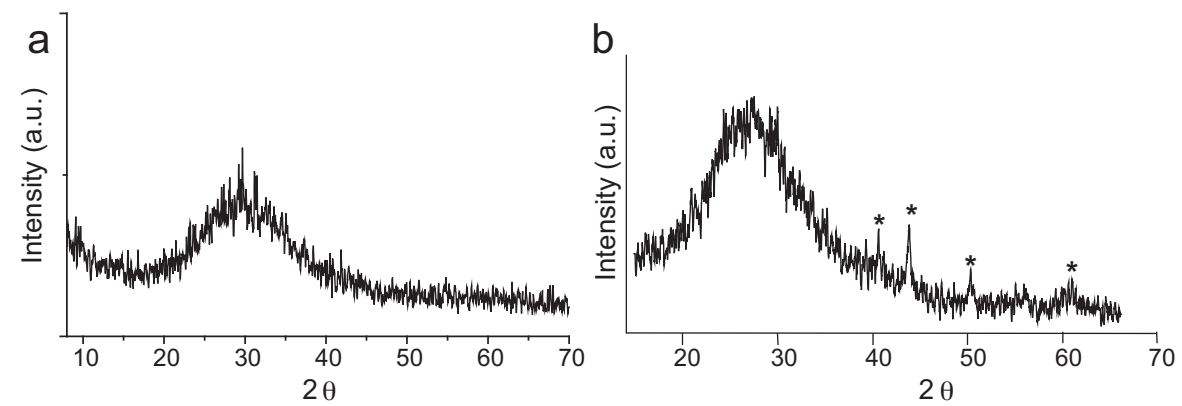

Fig. 5. XRD pattern for the $\mathrm{Cr}-\mathrm{MCM}-41$ (a) and $\mathrm{Cr}-\mathrm{SiO}_{2} /$ sol-gel (b) samples. ${ }^{*} \mathrm{Cr}_{2} \mathrm{O}_{3}$. 
Table 2

Oxidation of $\beta$-pinene (1) catalyzed by $\mathrm{Cr}$-catalysts under solvent-free conditions. ${ }^{\text {a }}$

\begin{tabular}{|c|c|c|c|c|c|c|c|c|c|c|}
\hline \multirow[t]{2}{*}{ Run } & \multirow[t]{2}{*}{ Catalyst } & \multirow[t]{2}{*}{$P($ atm $)$} & \multirow[t]{2}{*}{ Time $(\mathrm{h})$} & \multirow[t]{2}{*}{ Conversion (\%) } & \multicolumn{3}{|c|}{ Product selectivity (\%) } & \multirow[b]{2}{*}{7} & \multirow[t]{2}{*}{$S_{\text {allyl }}^{\mathrm{b}}(\%)$} & \multirow[t]{2}{*}{$\mathrm{TON}^{\mathrm{c}}$} \\
\hline & & & & & 4 & 5 & 6 & & & \\
\hline 1 & Cr-MCM-41 & 1 & 6 & 28 & 29 & 24 & 18 & 21 & 92 & 76 \\
\hline 2 & Cr-MCM-41 & 5 & 3.5 & 32 & 26 & 22 & 15 & 17 & 80 & 87 \\
\hline $3^{d}$ & Cr-MCM-41 & 1 & 6 & 4 & - & - & - & - & - & 11 \\
\hline $4^{e}$ & Cr-MCM-41 & 1 & 6 & 26 & 28 & 22 & 18 & 22 & 90 & 146 \\
\hline $5^{f}$ & Cr-MCM-41 & 1 & 6 & 28 & 30 & 22 & 16 & 23 & 91 & 222 \\
\hline 6 & $\mathrm{Cr}-\mathrm{SiO}_{2} /$ sol-gel & 1 & 6 & 29 & 30 & 14 & 14 & 16 & 74 & 78 \\
\hline 7 & $\mathrm{Cr}-\mathrm{SiO}_{2} /$ sol-gel & 5 & 3.5 & 30 & 18 & 16 & 9 & 10 & 53 & 81 \\
\hline
\end{tabular}

a Conditions: catalyst ( $3.5 \mathrm{wt} \%), 60^{\circ} \mathrm{C}$, gas phase $\mathrm{O}_{2}$, reaction time $6.0 \mathrm{~h}$ at $1 \mathrm{~atm}$ and $3.5 \mathrm{~h}$ at 5 atm. Conversion and selectivity were determined by $\mathrm{GC}$.

b Selectivities for allylic oxidation products 4-7.

c TON-moles of the substrate converted/moles of $\mathrm{Cr}$.

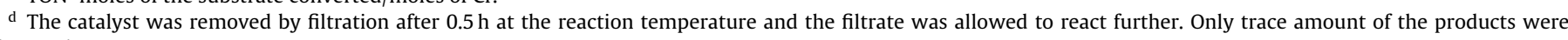
detected.

e The catalyst was re-used after run 1; total TON for two reaction cycles is given.

f The catalyst was re-used after run 4; total TON for three reaction cycles is given.

Table 3

Oxidation of $\alpha$-pinene (2) catalyzed by $\mathrm{Cr}$-catalysts under solvent-free conditions. ${ }^{\text {a }}$

\begin{tabular}{|c|c|c|c|c|c|c|c|c|c|c|c|}
\hline \multirow[t]{3}{*}{ Run } & \multirow[t]{3}{*}{ Catalyst } & \multirow[t]{3}{*}{$P(\mathrm{~atm})$} & \multirow[t]{3}{*}{ Conversion (\%) } & \multicolumn{5}{|c|}{ Product selectivity (\%) } & \multirow[t]{3}{*}{$S_{\text {allyl }}{ }^{\mathrm{b}}(\%)$} & \multirow[t]{3}{*}{$S_{\text {epox }}{ }^{\mathrm{b}}(\%)$} & \multirow[t]{3}{*}{$\mathrm{TON}^{\mathrm{c}}$} \\
\hline & & & & \multicolumn{3}{|c|}{ Allylic oxidation } & \multicolumn{2}{|c|}{ Epoxidation } & & & \\
\hline & & & & 4-7 & 8 & 9 & 10 & 11 & & & \\
\hline 1 & Cr-MCM-41 & 1 & 40 & 12 & 33 & 26 & 8 & 4 & 71 & 12 & 108 \\
\hline 2 & Cr-MCM-41 & 5 & 38 & 13 & 25 & 26 & 5 & 3 & 64 & 8 & 103 \\
\hline 3 & $\mathrm{Cr}-\mathrm{SiO}_{2} /$ sol-gel & 1 & 45 & 10 & 22 & 16 & 8 & 3 & 48 & 11 & 122 \\
\hline 4 & $\mathrm{Cr}-\mathrm{SiO}_{2} /$ sol-gel & 5 & 37 & 8 & 25 & 12 & 3 & 3 & 45 & 6 & 100 \\
\hline
\end{tabular}

a Conditions: catalyst ( $3.5 \mathrm{wt} \%), 60^{\circ} \mathrm{C}$, gas phase $\mathrm{O}_{2}$, reaction time $6.0 \mathrm{~h}$ at $1 \mathrm{~atm}$ and $3.5 \mathrm{~h}$ at 5 atm. Conversion and selectivity were determined by GC.

b Selectivities for allylic oxidation and epoxidation products: 4-9 and $\mathbf{1 0}+\mathbf{1 1}$, respectively.

c $\mathrm{TON}-$ moles of the substrate converted/moles of $\mathrm{Cr}$.

should be considered a good to excellent result for radical reactions. At the oxidation of $\alpha$-pinene, two major products accounted for $50-60 \%$ of the mass balance. $\beta$-Pinene gave almost exclusively the products of allylic oxidation, i.e., allylic ketones and alcohols; whereas limonene and $\alpha$-pinene also produced epoxides in significant amounts.

One of the technological advantages of the process is a possibility to obtain high concentrations of valuable oxygenated products in the final mixtures (30-40wt\%) as the reactions occur in the absence of solvent. Although several major products are formed from each substrate, for practical purposes their separation is often not necessary as the mixtures themselves show interesting organoleptic properties and can be used directly in fragrance compositions. Moreover, even the separation of the unreacted substrate from the products is often not necessary because the oils enriched in oxygenated compounds find various direct practical applications and cost much more than original oils with higher content of nonfunctionalized terpenes.

As it will be shown below, the Cr-MCM-41 catalyst does not release the active metal into the solution and can be removed from the reaction mixture by simple centrifugation or filtration. Such a simple handling represents another attractive feature of the process. The recovered catalyst after washing can be re-used several times without a significant loss of activity and selectivity.

The oxidation of $\beta$-pinene (1) in the presence of $\mathrm{Cr}-\mathrm{MCM}-41$ occurred smoothly resulting in a $28 \%$ conversion for $6 \mathrm{~h}$ at $1 \mathrm{~atm}$ of oxygen (Table 2 , run 1 ). The reaction gave almost exclusively allylic oxidation products, i.e., trans-pinocarveol (4), pinocarvone (5), myrtenal (6), and myrtenol (7), with a high combined selectivity of $92 \%$ based on the reacted substrate (Scheme 1 ). No epoxide or epoxide derived products were detected in the reaction mixtures. In our previous studies, $\beta$-pinene also exhibited a strong preference for the allylic oxidation over epoxidation, which was explained by the enhanced reactivity of the allylic hydrogen [26,27]. The results of run 1 in Table 2 corresponded to a turnover number of 76 with respect to the total amounts of chromium in the material. However, considering that most of the chromium ions were located in the bulk solid and were not accessible to the substrate, the real efficiency of the surface chromium species was much higher. In other words, if only the surface chromium species could be counted, the

Table 4

Oxidation of limonene (3) catalyzed by $\mathrm{Cr}$-catalysts under solvent-free conditions. ${ }^{\text {a }}$

\begin{tabular}{|c|c|c|c|c|c|c|c|c|c|c|}
\hline \multirow[t]{3}{*}{ Run } & \multirow[t]{3}{*}{ Catalyst } & \multirow[t]{3}{*}{$P($ atm $)$} & \multirow[t]{3}{*}{ Conversion (\%) } & \multicolumn{4}{|c|}{ Product selectivity (\%) } & \multirow[t]{3}{*}{$S_{\text {allyl }}{ }^{\mathrm{c}}(\%)$} & \multirow[t]{3}{*}{$S_{\text {epox }}{ }^{\mathrm{c}}(\%)$} & \multirow[t]{3}{*}{$\mathrm{TON}^{\mathrm{d}}$} \\
\hline & & & & \multicolumn{3}{|c|}{ Allylic oxidation } & \multirow{2}{*}{$\frac{\text { Epoxidation }^{\mathrm{b}}}{\mathbf{1 5}}$} & & & \\
\hline & & & & 12 & 13 & 14 & & & & \\
\hline 1 & Cr-MCM-41 & 1 & 36 & 22 & 8 & 25 & 20 & 55 & 20 & 97 \\
\hline 2 & Cr-MCM-41 & 5 & 41 & 17 & 6 & 22 & 17 & 45 & 17 & 111 \\
\hline 3 & $\mathrm{Cr}-\mathrm{SiO}_{2} /$ sol-gel & 1 & 49 & 12 & 4 & 13 & 19 & 29 & 19 & 132 \\
\hline 4 & $\mathrm{Cr}-\mathrm{SiO}_{2} /$ sol-gel & 5 & 46 & 12 & 3 & 10 & 13 & 25 & 13 & 124 \\
\hline
\end{tabular}

a Conditions: catalyst ( $3.5 \mathrm{wt} \%), 60^{\circ} \mathrm{C}$, gas phase $\mathrm{O}_{2}$, reaction time $6.0 \mathrm{~h}$ at $1 \mathrm{~atm}$ and $3.5 \mathrm{~h}$ at 5 atm. Conversion and selectivity were determined by GC.

b Mainly endo epoxide 15, along with small amounts of exo epoxide (not shown); endo/exo $\approx 4 / 1$.

c Selectivities for allylic oxidation and epoxidation products: 12-14 and 15 + exo epoxide, respectively.

d TON-moles of the substrate converted/moles of $\mathrm{Cr}$. 


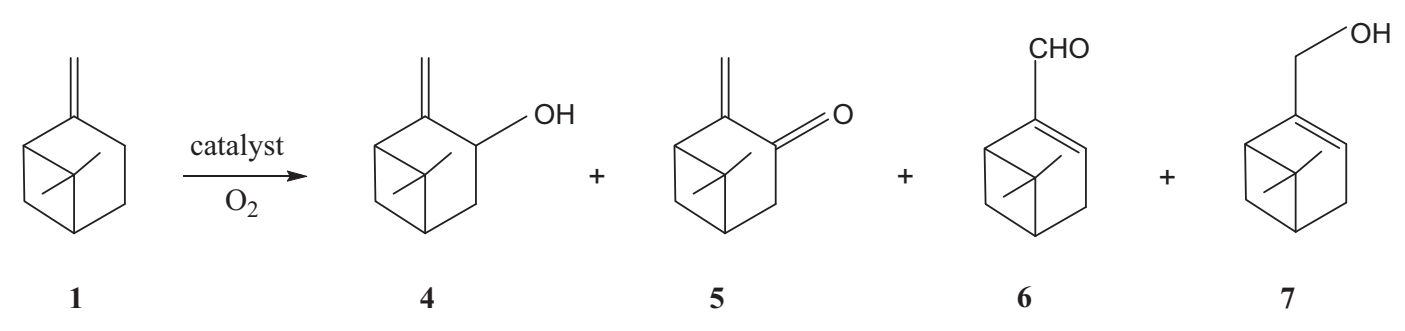

Scheme 1. Oxidation of $\beta$-pinene (1).

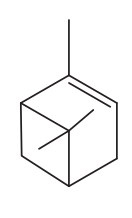

2

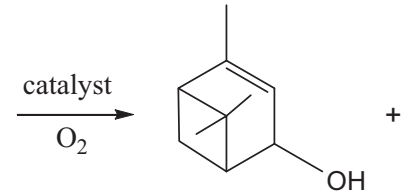

8

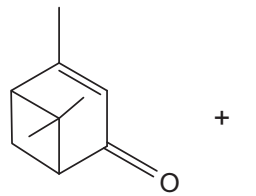

9

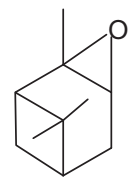

10

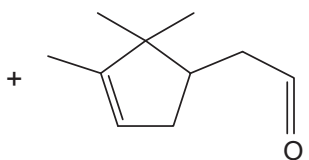

11

Scheme 2. Oxidation of $\alpha$-pinene (2).

TON would be higher. It should be mentioned that the reaction rate was not affected by changes in the intensity of stirring, thus the diffusion limitations, if exist, seem to be intraparticle ones.

The reaction was faster under 5 atm of oxygen than at atmospheric pressure; however, selectivity was lower (cf. run 2 and run 1 in Table 2). Similar results were obtained for limonene and $\alpha$ pinene (Tables 3 and 4). Thus, there is no practical advantage in using elevated oxygen pressures for this process, which obviously would involve the use of more expensive equipments and more rigorous safety requirements.

The leaching of chromium ions under the reaction conditions was verified in special experiments. In run 3 (Table 2), the $\mathrm{Cr}$ MCM-41 catalyst was filtered off during the course of the reaction at the reaction temperature to avoid re-adsorption of leached metal ions onto the solid support. Then, the filtrate was allowed to react further. No further conversion of $\beta$-pinene was observed after catalyst removing, providing strong evidence in support of heterogeneous catalysis. Thus, the reaction solution contained no significant amounts of dissolved chromium species and the activity of the catalyst was due to the chromium ions immobilized in the mesoporous silica framework.

After the reaction in run 1 (Table 2 ), the catalyst was removed by centrifugation, washed with acetonitrile and ethanol and reused two times (Table 2, runs 4 and 5). The behavior of the spent catalyst with fresh substrate was near the same as in the original reaction. A total TON for three reaction cycles reached the value of 222 . Thus, the catalyst released no chromium into the medium and can be easily recovered either by centrifugation or filtration and reused. The results obtained in leaching and recycling experiments are in agreement with previous studies $[16,18,19]$ reporting that MCM-41-included chromium catalysts do not suffer metal leaching during the oxidation reactions. In some cases $[17,20]$ the catalysts lost the activity but only in the first recycling experiment due to leaching of non-framework chromium ions and then their activity was stable.

The oxidation of $\alpha$-pinene (2) over the Cr-MCM-41 catalyst gave mainly verbenol (8) (ca. 80\% trans) and verbenone (9) (25-30\% each) as well as small amounts of $\alpha$-pinene oxide (10) and campholenic aldehyde (11) (Scheme 2, Table 3). Campholenic aldehyde more likely resulted from the skeletal rearrangement of $\alpha$-pinene oxide [36], thus it was referred in Table 2 as the epoxidation product. In addition, the products of the allylic oxidation of $\beta$ pinene (4-7) were observed in appreciable amounts indicating that the partial isomerization of $\alpha$-pinene occurred in the reaction medium. On the other hand, no products derived from $\alpha$-pinene were detected at the oxidation of $\beta$-pinene over the Cr-MCM-41 catalyst. The same substrate conversion of nearly $40 \%$ was achieved under 5 atm of oxygen for shorter time; however, the product selectivity slightly decreased (Table 3 , run 1 vs. run 2 ).

The reaction with limonene (3) over Cr-MCM-41 resulted in three main products: carveol (12) (ca. 80\% cis), carvone (14), and limonene oxide (15) (cis/trans $\approx 1 / 1$ ), each at $20-25 \%$ selectivity (Scheme 3, Table 4). Approximately $40 \%$ conversion was attained for $6 \mathrm{~h}$ under $1 \mathrm{~atm}$ and for $3.5 \mathrm{~h}$ under $5 \mathrm{~atm}$ of oxygen; however, the selectivities for each of the identified products 12-15 were lower, likewise with $\alpha$-pinene and $\beta$-pinene (Table 4 , runs 1 and 2). The endo cyclic double bond of limonene was much more sensitive to epoxidation as the epoxide resulting from the oxidation of the exo cyclic double bond (not shown) was detected only in small amounts. A higher reactivity of the endo cyclic double bond of limonene compared to the exo cyclic one toward allylic oxidation is due to a so-called "cyclic activation", which is an

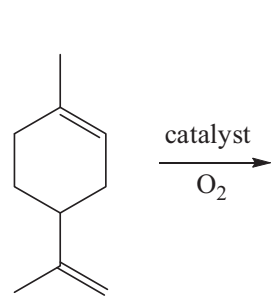

3<smiles>C=C(C)C1CC=C(C)C(O)C1</smiles>

12<smiles>C=C(C)C1CC=C(CO)CC1</smiles>

13<smiles>C=C(C)C1CC=C(C)C(=O)C1</smiles>

14<smiles>C=C(C)C1CCC2(C)OC2C1</smiles>

15

Scheme 3. Oxidation of limonene (3). 
enhanced reactivity of cyclic allylic hydrogens compared to acyclic ones [27].

It should be mentioned that, in the presence of chromium catalysts, both limonene and $\alpha$-pinene have demonstrated a stronger preference to allylic oxidation over epoxidation as compared to cobalt catalysts. In our previous works $[25,27]$ a molar ratio between allylic oxidation and epoxidation products at the oxidation of limonene and $\alpha$-pinene over the cobalt catalysts varied from $1 / 1$ to $2 / 1$, whereas the $\mathrm{Cr}-\mathrm{MCM}-41$ catalyst produced allylic products in much higher proportions (3/1-6/1). Thus, chromium catalysts represent interesting alternative for the conventional cobalt catalysts in the aerobic oxidations of essential oil-based alkenes; in addition, they could result in fragrance mixtures with other organoleptic characteristics.

The $\mathrm{Cr}-\mathrm{SiO}_{2} /$ sol-gel material also promoted the oxidation of all three substrates, $\beta$-pinene, limonene and $\alpha$-pinene. Product distributions were similar to those obtained with $\mathrm{Cr}-\mathrm{MCM}-41$; however, the reaction selectivity was significantly lower. The combined selectivity for products 4-7 formed from $\beta$-pinene decreased from $92 \%$ with the Cr-MCM-41 catalyst to $74 \%$ with the conventional catalyst prepared without the surfactant, $\mathrm{Cr}-\mathrm{SiO}_{2} /$ sol-gel (Table 2, run 1 vs. run 6). Similar results were obtained at the oxidation of $\alpha$-pinene (Table 3, run 1 vs. run 3 ) and limonene (Table 4 , run 1 vs. run 3 ), where the combined selectivity for the identified products decreased from 83 to $59 \%$ and from 75 to $48 \%$, respectively.

Although the surface area of the Cr-MCM-41 material is two and a half times higher than that of $\mathrm{Cr}-\mathrm{SiO}_{2} /$ sol-gel (Table 1 ), their activities were quite similar. Thia could be related to the fact that in the $\mathrm{Cr}-\mathrm{SiO}_{2} / \mathrm{sol}$-gel a larger proportion of chromium might be accessible for the substrate due to the larger amounts of extraframework $\mathrm{Cr}_{2} \mathrm{O}_{3}$ or/and less amounts of small pores.

In the previous work [5], we have observed a remarkably high selectivity of the MCM-41-included cobalt catalyst in the oxidation of isolongifolene. The activity of Co-MCM- 41 was much higher than that of the material prepared by the conventional sol-gel method without the surfactant. The results obtained in the present study clearly show that a highly organized structure of the $\mathrm{Cr}$ MCM-41 material also exerts a pronounced beneficial effect on the selectivity of the oxidation of $\beta$-pinene, limonene, and $\alpha$-pinene. In spite of the $\mathrm{Cr}-\mathrm{SiO}_{2} /$ sol-gel catalyst having larger pores and higher total pore volume compared to $\mathrm{Cr}-\mathrm{MCM}-41$, the reactions over $\mathrm{Cr}-\mathrm{SiO}_{2} /$ sol-gel were less selective. This implies that pore geometry is a crucial factor to ensure high selectivity of chromium species during the reaction and to avoid over-oxidation. The structure of the solid matrix should allow a rapid diffusion of bulky products throughout the porous medium to prevent successive reactions. A highly ordered uniform channel-like pore geometry and facile pore connectivity in the $\mathrm{Cr}-\mathrm{MCM}-41$ material should favor the transport of the products away from the active chromium sites. The $\mathrm{Cr}-\mathrm{SiO}_{2}$ /sol-gel material, on the other hand, shows much more heterogeneous pore shape distribution and might posses ink-bottle pores with narrow mouths. Thus, the primarily formed products could undergo a further oxidation in this material due to a longer contact with catalytically active sites. Besides, the chromium active sites in $\mathrm{Cr}-\mathrm{MCM}-41$ are expected to be more energetically homogeneous than those in $\mathrm{Cr}-\mathrm{SiO}_{2} /$ sol-gel, which shows lower surface uniformity. Thus, the textural properties of the Cr-MCM-41 material remarkably benefit the overall selectivity of the oxidation reactions.

\section{Conclusions}

In summary, chromium-incorporated molecular sieves are efficient catalysts for the aerobic oxidation of monoterpenic alkenes under mild conditions. The use of the renewable biomass-based compounds as the substrates, chromium immobilized in a solid matrix as the catalyst, molecular oxygen as the final oxidant, and solvent free conditions are significant practical advantages of this environmentally friendly process. The catalyst undergoes no metal leaching, and can be easily recovered and re-used without a special treatment. The Cr-MCM-41 material exhibited better performance than the sample prepared by the conventional sol-gel method without the surfactant. Further studies are directed toward the applications of chromium-incorporated ordered mesoporous molecular sieves for the oxidation of other renewable substrates.

\section{Acknowledgments}

Financial support from the CNPq, CAPES, FAPEMIG and INCTCatálise (Brazil) is gratefully acknowledged. The authors wish to thank the Microscopy Center, UFMG, Brazil and the LNLS synchrotron laboratory, Campinas, Brazil for the equipment time.

\section{References}

[1] I.W.C.E. Arends, R.A. Sheldon, Appl. Catal., A 212 (2001) 175-187.

[2] M. Ziolek, Catal. Today 90 (2004) 145-150.

[3] C.T. Kresge, M.E. Leonowicz, W.J. Roth, J.C. Vartuli, J.S. Beck, Nature 359 (1992) $710-712$.

[4] J.S. Beck, J.C. Vartuli, W.J. Roth, M.E. Leonowicz, C.T. Kresge, K.C. Chu, D.H. Olson, E.W.Sheppard, S.B. McCullen, J.L. Schenkler, J. Am. Chem. Soc. 114(1992) 10834-10843.

[5] P.A. Robles-Dutenhefner, K.A. da Silva Rocha, E.M.B. Sousa, E.V. Gusevskaya, J. Catal. 265 (2009) 72-79.

[6] J. Muzart, Chem. Rev. 92 (1992) 113-140.

[7] B.M. Choudary, A.D. Prasad, V. Swapna, V.L.K. Valli, V. Bhuma, Tetrahedron 48 (1992) 953-962.

[8] H.E.B. Lempers, R.A. Sheldon, Appl. Catal., A 143 (1996) 137-143.

[9] W.A. Carvalho, P.B. Varaldo, M. Wallau, U. Schuchardt, Zeolites 18 (1997) 408-416.

[10] S. Shylesh, Ch. Srilakshmi, A.P. Singh, B.G. Anderson, Microporous Mesoporous Mater. 99 (2007) 334-344.

[11] J.D. Chen, R.A. Sheldon, J. Catal. 153 (1995) 1-8.

[12] I.C. Chisem, J. Rafelt, J. Chisem, J.H. Clark, D. Macquarrie, M.T. Shieh, R. Jachuck, C. Ramshaw, K. Scott, Chem. Commun. (1998) 1949-1950.

[13] S. Shylesh, P.P. Samuel, A.P. Singh, Appl. Catal., A 318 (2007) 128-136.

[14] W. Zhang, J. Wang, P.T. Tanev, T.J. Pinnavaia, Chem. Commun. (1996) 979-980.

[15] N. Ulagappan, C.N.R. Rao, Chem. Commun. (1996) 1047-1048.

[16] T.K. Das, K. Chaudhari, E. Nandanan, A.J. Chandwadkar, A. Sudalai, T. Ravindranathan, S. Sivasanker, Tetrahedron Lett. 38 (1997) 3631-3634.

[17] A. Sakthivel, S.K. Badamali, P. Selvam, Catal. Lett. 80 (2002) 73-76.

[18] N. Srinivas, V. Radha Rani, S.J. Kulkarni, K.V. Raghavan, J. Mol. Catal. A 179(2002) 221-231.

[19] S. Samanta, N.K. Mal, A. Bhaumik, J. Mol. Catal. A 236 (2005) 7-11.

[20] A. Sakthivel, P. Selvam, J. Catal. 211 (2002) 134-143.

[21] H. Mimoun, Chimia 50 (1996) 620-625.

[22] M.J. da Silva, J.A. Gonçalves, O.W. Howarth, R.B. Alves, E.V. Gusevskaya, J Organomet. Chem. 689 (2004) 302-308.

[23] J.A. Gonçalves, M.J. da Silva, D. Piló-Veloso, O.W. Howarth, E.V. Gusevskaya, J Organomet. Chem. 690 (2005) 2996-3003.

[24] M.G. Speziali, P.A. Robles-Dutenhefner, E.V. Gusevskaya, Organometallics 26 (2007) 4003-4009.

[25] M.J. da Silva, P.A. Robles-Dutenhefner, L. Menini, E.V. Gusevskaya, J. Mol. Catal. A 201 (2003) 71-77.

[26] L. Menini, M.J. da Silva, M.F.F. Lelis, J.D. Fabris, R.M. Lago, E.V. Gusevskaya, Appl Catal., A 269 (2004) 117-121.

[27] L. Menini, M.C. Pereira, L.A. Parreira, J.D. Fabris, E.V. Gusevskaya, J. Catal. 254 (2008) 355-364.

[28] E.F. Murphy, T. Mallat, A. Baiker, Catal. Today 57 (2000) 115-126.

[29] Y. Shiraishi, Y. Teshima, T. Hirai, J. Phys. Chem. B 110 (2006) 6257-6263.

[30] F. Amano, T. Yamaguchi, T. Tanaka, J. Phys. Chem. B 110 (2006) 281-288.

[31] M. Kruk, M. Jaroniec, Chem. Mater. 13 (2001) 3169-3183.

[32] A. Corma, Chem. Rev. 97 (1997) 2373-2419.

[33] A.B. Gaspar, R.L. Martins, M. Schmal, L.C. Dieguez, J. Mol. Catal. A 169 (2001) $105-112$.

[34] P. Michorczyk, J. Ogonowski, P. Kuš̌trowski, L. Chmielarz, Appl. Catal., A 349 (2008) 62-69.

[35] T.V.M. Rao, Y. Yang, A. Sayari, J. Mol. Catal. A 301 (2009) 152-158.

[36] K.A. da Silva Rocha, J.L. Hoehne, E.V. Gusevskaya, Chem. Eur. J. 14 (2008) 6166-6172. 